\title{
El déficit comercial de Estados Unidos frente a la entrada de China a su mercado
}

\section{Resumen}

Este artículo analiza las causas del déficit comercial de Estados Unidos (DCEU), desde la perspectiva macroeconómica y estructural, a partir de la década de los noventa, sin abarcar los años de la última crisis financiera del siglo XXI, que debilitó el crecimiento del DCEU. Una vez establecido el marco de referencia, el texto estudia la coyuntura que marca el desplazamiento de Japón por China, como la relación comercial deficitaria más importante para Estados Unidos, para enfocarse en las posibles causas de la creciente participación de China en el DCEU. Finalmente, este documento presenta un comparativo de los índices de ventaja comparativa revelada entre el Este de Asia, China, y Estados Unidos, con el objetivo de exponer una de las causas estructurales más importantes del DCEU.

Palabras clave: Estados Unidos, Este de Asia, China, déficit comercial, ventaja comparativa.

\section{Abstract}

The primary focus of this article is to analyze the causes of U.S. trade deficit throughout the last two decades. This deficit is studied from the macroeconomic and structural perspective, without covering the years of the last century financial crisis that weakened the growth of U.S. trade deficit. This article

1. Artículo recibido el 19 de julio de 2012 y dictaminado el 03 de septiembre de 2012. Ximena Valentina Echenique Romero: profesora de El Colegio de la Frontera Norte.

ORCID http://orcid.org/0000-0002-5485-3282 
exhibits the shift from Japan to China as the most imbalanced bilateral trade relationship for the United States, focusing on the possible causes of China's increasing participation in U.S. trade deficit. These facts are classified into exogenous and endogenous in order to establish the uniqueness of China's presence in the U.S. market. Finally, this paper presents a comparison of revealed comparative advantage indices among the major economies of East Asia, China and the United States to expose the regional dynamics that helps China to remain a leading exporter.

Keywords: The United States, East Asia, China, trade deficit, comparative advantage.

\section{Introducción}

En términos macroeconómicos, el estudio del déficit comercial (DC) de un país comienza con la identidad contable que iguala el saldo de la cuenta corriente, que determina el superávit o déficit externo de una nación, con la diferencia entre el ahorro y la inversión. En razón a la igualdad 3.1 (recuadro 1), el monto de inversiones domésticas por arriba del ahorro nacional es la causa principal del DC de un país y el financiamiento de esta diferencia requiere de entradas de capital foráneo y ubica a la nación deficitaria como un prestatario internacional neto. Este enfoque no aporta una teoría sobre las causas económicas subyacentes del desequilibrio macroeconómico, por lo que, la identidad contable es una herramienta descriptiva y no analítica del tema que me ocupa.

La visión estructural, que adopta este artículo como marco teórico, liga el DC con un conjunto de prácticas comerciales, políticas industriales y apoyos gubernamentales que forman parte de los spillover o incidencias alrededor del sistema comercial internacional, incluidas las de orden económico, político y social. En general, se trata de un conjunto de factores que, entre otras variables, están manifiestos en los índices de ventaja comparativa revelada como se observará en la parte final de este texto.

En términos macroeconómicos, este artículo argumenta que el DCEU ha sido resultado de una política económica dirigida a financiar el crecimiento de Estados Unidos a través de ahorros foráneos. Respecto a la visión estructural, el presente texto sostiene que un proceso regional de reciclaje de ventaja comparativa en el Este de Asia (Corea del Sur (Corea), Taiwán, Hong Kong, Indonesia, Malasia, Filipinas, Tailandia, Singapur y Vietnam) y un centro 


\section{Recuadro 1}

\section{Identidad contable del déficit comercial}

El cálculo del PIB por el método del gasto permite igualar la balanza comercial con la diferencia entre el ahorro y la inversión de un país, en general, por ser la balanza comercial de mercancías el saldo determinante de la cuenta corriente. De acuerdo con el citado método, el Producto Interno Bruto $(\mathrm{Y})$ es la suma del consumo personal (C), el consumo gubernamental (G), la inversión (I) y la balanza comercial (BC): exportaciones (E) menos importaciones (IM).

1) $\quad \mathrm{Y}=\mathrm{C}+\mathrm{G}+\mathrm{I}+(\mathrm{E}-\mathrm{IM})$

1.1) $Y=C+G+I+B C$

2) $\quad B C=Y-[C+G+I]$

La ecuación 2) dice que la balanza comercial es igual a la diferencia entre ingreso doméstico y gasto nacional. Si el ingreso excede al gasto, hay una BC superavitaria, y viceversa. Reacomodando la ecuación 2) la balanza comercial es igual al ahorro nacional menos la inversión nacional.

3) $\mathrm{BC}=[\mathrm{Y}-\mathrm{C}-\mathrm{G}]-\mathrm{I}$
3.1) $\quad \mathrm{E}-\mathrm{IM}=\mathrm{A}-\mathrm{I}$;
A: Ahorro

$\mathrm{Al}$ existir una diferencia entre las operaciones del gobierno y aquellas del sector privado, la igualdad anterior queda definida como:

3.2) $\quad B C=\left[A^{P}+A^{G}\right]-I ; \quad A^{P:}$ Ahorro privado $A^{G}$ : Ahorro gubernamental

El ahorro privado, $\mathrm{A}^{\mathrm{P}}=\mathrm{Y}^{\mathrm{P}}-\mathrm{C}-\mathrm{T}$, es el ingreso privado (corporativo y personal) menos el consumo, $\mathrm{C}$, y el pago de impuestos, T. (T: tax, para evitar una confusión con el signo inversión I)

El ahorro gubernamental, $\mathrm{A}^{\mathrm{G}}=\mathrm{T}-\mathrm{G}$, es el ingreso gubernamental por impuestos, $\mathrm{T}$, menos el gasto de gobierno, $\mathrm{G}$.

de procesamiento exportador de alta tecnología operando en la República Popular China (China, en adelante) están entre las variables explicativas más importantes de la primacía de China en la composición por país del DCEU.

En la segunda parte, el artículo analiza el desplazamiento de Japón por China como la relación comercial deficitaria más significativa para Estados Unidos dentro de su tercera fase de relaciones comerciales con $\mathrm{China}^{2}$, cuya

2. La primera fase de restauración de las relaciones comerciales entre Estados Unidos y China comenzó en los primeros años de la década de los setenta, cuando la administración Nixon (1972) presionó por un proceso gradual de normalización de estas. La segunda etapa se 
importancia da cuenta el hecho de que las exportaciones chinas hacia Estados Unidos representaron un máximo de 45\% del DCEU en 2009.

\section{Una lectura macroeconómica del déficit comercial de Estados Unidos}

En Estados Unidos, como en la mayoría de los países, la diferencia entre exportaciones e importaciones de bienes físicos o mercancías es el principal componente de la cuenta corriente. Hasta el 2009, este rubro fue seguido por la balanza en servicios. Aunque, recientemente, el saldo de los ingresos sobre inversiones y las transferencias unilaterales han cobrado mayor importancia en la cuenta corriente (gráfica 1).

\section{Gráfica 1}

Estructura de la cuenta corriente de Estados Unidos

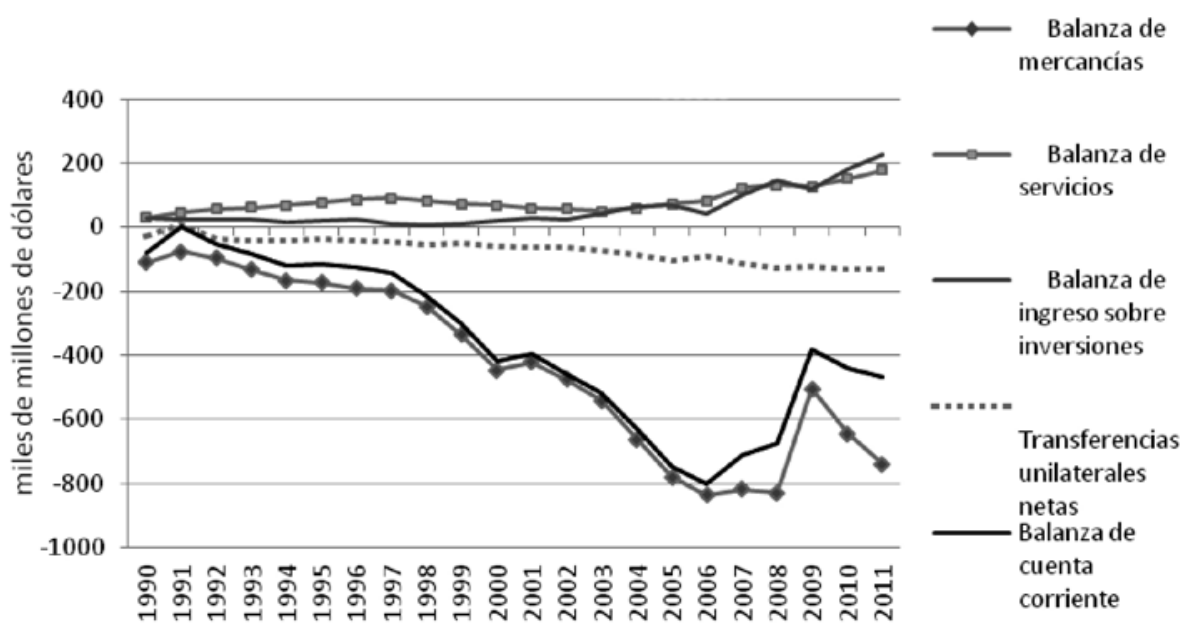

Fuente: Bureau of Economic Analysis. www.bea.gov/index.htm, consultado septiembre 6, 2012.

inició en 1979, con las reformas económicas en China y la extensión del Trato de Nación Más Favorecida por parte de Estados Unidos (1979), la cual estuvo sujeta a una dispensa presidencial anual hasta la incorporación de China a la OMC en 2001. 
El DCEU alcanzó una participación máxima sobre el PIB estadounidense de $6.25 \%$ en 2006. Entre el tercer trimestre del 2008 y el primer trimestre del 2009, el DCEU mantuvo una caída significativa como resultado de la recesión económica estadounidense y, desde entonces, ha presentado una recuperación de su tendencia positiva de carácter estructural.

Desde la identidad macroeconómica contable, desglosada en la introducción, el déficit comercial es un problema macroeconómico a largo plazo de desahorro nacional, que ha estado presente en la economía de Estados Unidos desde la década de los ochenta del siglo pasado. Durante el Reaganomics ${ }^{3}$ (1981-1989), el desahorro gubernamental en Estados Unidos fue resultado del fuerte recorte impositivo, el crecimiento de la deuda pública y el aumento del gasto militar.

A finales de los años ochenta, el balance presupuestal de Estados Unidos comenzó a mejorar considerablemente por un conjunto de políticas económicas de oferta, logrando cuatro años de superávit fiscal al cierre de la década de los noventa, los cuales contribuyeron positivamente sobre el ahorro estadounidense $e^{4}$. Sin embargo, durante los últimos años de la citada década, la esfera privada siguió el comportamiento opuesto, la inversión creció mientras el ahorro descendió, presentándose un escenario de desahorro nacional frente al ciclo de crecimiento económico asociado al desarrollo tecnológico (proceso referido como "burbuja de la nueva economía"5). En los primeros años del nuevo milenio, el ahorro estadounidense, tanto privado como gubernamental, descendió en sinergia con el crecimiento del mercado inmobiliario (tabla 1).

3. Este término se refiere al conjunto de políticas económicas que condujeron a un creciente déficit presupuestal y altas tasas de interés reales durante la administración Reagan.

4. En 1983 el déficit fiscal de Estados Unidos representó el 6\% de su PIB. En 1997 el déficit presupuestal alcanzó un nivel mínimo de $0.3 \%$ sobre el PIB estadounidense, seguido por cuatro años de saldos positivos, alcanzando un máximo de 2.4\% en 2000 (BEA, National Economic Accounts, 2010).

5. El término burbuja explica el proceso donde los precios y los dividendos de los valores no guardan relación con los índices de producción, productividad y capitalización real del sector en cuestión (Pérez, 2002). Para una detallada comprensión de las burbujas punto com e inmobiliaria, desarrolladas en Estados Unidos durante la dirección de Alan Greenspan en la Reserva Federal de Estados Unidos, véase: Krugman, 2009: 139-152. 


\section{Tabla 1}

El déficit comercial estadounidense desde

la identidad macroeconómica contable

\begin{tabular}{lll}
\hline Años ochenta & Finales de los años noventa & Principios del nuevo milenio \\
\hline$(\mathrm{A}-\mathrm{I})+(\mathrm{T}-\mathrm{G}-\mathrm{TR})=(\mathrm{E}-\mathrm{IM})$ & $(\mathrm{A}-\mathrm{I})+(\mathrm{T}-\mathrm{G}-\mathrm{TR})=(\mathrm{E}-\mathrm{IM})$ & $(\mathrm{A}-\mathrm{I})+(\mathrm{T}-\mathrm{G}-\mathrm{TR})=(\mathrm{E}-\mathrm{IM})$ \\
$(-)(-)(-)$ & $(-)(+)(-)$ & $(-)(-)(-)$
\end{tabular}

A: Ahorro

I: Inversión

T: Impuestos

G: Gasto de gobierno

TR: Transferencias

E: Exportaciones

IM: Importaciones

El desajuste entre los fondos domésticos disponibles y la inversión local condujeron el cambio de Estados Unidos, de la más grande nación acreedora a la mayor nación deudora a escala mundial. A partir de 1983, la posición de la inversión internacional neta estadounidense ha sido continuamente negativa y equivalente al excedente de inversión en capital respecto a los ahorros nacionales. El capital foráneo ingresado en la cuenta de capital ha financiado el desequilibrio macroeconómico estadounidense y ha permitido a los ciudadanos de ese país comprar más de lo que venden en el mercado internacional de bienes y servicios, además de hacer posible un crecimiento económico mayor en relación al que permitirían los recursos locales.

El efecto ingreso y de precios relativos en el déficit comercial de Estados Unidos

Los flujos de inversión extranjera interactúan con el desequilibrio macroeconómico nacional entre ahorro e inversión a través del efecto ingreso y el efecto de precios relativos, y ambos determinan la dinámica del déficit externo (Blacker, 1992; L. Mann, 1999). En Estados Unidos, durante 1992-99, el desarrollo de nuevas tecnologías en el procesamiento de la información y las telecomunicaciones (shock de oferta de largo plazo sobre el crecimiento), el avance de los mercados financieros, y el descenso de los precios de algunas importaciones clave para Estados Unidos, específicamente del petróleo (shock de oferta de corto plazo sobre el crecimiento), contribuyeron a la construcción de un excepcional ciclo expansivo de la economía estadounidense. Este perio- 
do coincidió con el despegue económico de China, la recesión económica de Japón y el descenso del ingreso en los países afectados por la crisis financiera del Este de Asia (1997), Rusia y Brasil (1999).

Durante 1996 y 2000, el conjunto de hechos antes referidos hicieron del vecino país del norte un atractivo destino para la inversión extranjera ${ }^{6}$. Las entradas netas de capital en Estados Unidos pasaron, de un promedio anual de \$105 mil millones de dólares en el periodo 1993-96, a poco más de \$174 mil millones de dólares por año entre 1997 y 1999. En 2000 estos flujos sumaron $\$ 478$ mil millones de dólares. Este ingreso significativo de capital foráneo capitalizó el mercado accionario de Estados Unidos e incrementó los precios de los títulos estadounidenses y el valor del dólar. Entre 1995 y 1999, el valor real del dólar aumentó de un índice de 86.85 a 100.97, y la proporción del DC sobre el PIB ascendió de 2.35\% a 3.75\% (BEA, National Economic Accounts, 2010). Porque la mayor riqueza derivada de este proceso aumentó el consumo estadounidense, incluyendo importaciones y desincentivó el ahorro nacional privado.

Entre 2001 y 2002, Estados Unidos presentó un nuevo ciclo económico decreciente. La inversión, tanto nacional como extranjera, en nuevo capital y la demanda por financiamiento externo disminuyeron como consecuencia del colapso del mercado de valores tecnológicos sucedido en marzo del año 2000. Sin embargo, los ahorros globales permanecieron fuertes y la inflación controlada, hecho que contribuyó al descenso de la tasa de interés real en Estados Unidos, el Reino Unido, Francia y Japón. Las bajas tasas de interés reales asociadas con el precio de los bonos gubernamentales estadounidenses indexados a la inflación limitaron las tasas de ahorros en Estados Unidos, ${ }^{7} \mathrm{y}$ fortalecieron la inversión en el mercado residencial estadounidense.

De 2003 a 2007, la economía estadounidense se desarrolló satisfactoriamente, en el grado de ser denominado un periodo expansivo (2003-2007), pero la dependencia de Estados Unidos respecto del capital extranjero aumentó considerablemente. Porque sus bajas tasas de interés reales, junto con su presupuesto federal deficitario y su apalancamiento financiero sobre el boom inmobiliario, fortalecieron el bajo nivel de ahorro nacional privado.

6. De acuerdo con Bernanke (2005), durante la década de los noventa, el bajo riesgo político, los estrictos derechos de propiedad intelectual y la adecuada regulación ambiental fueron elementos importantes en la atracción de flujos financieros externos hacia Estados Unidos.

7. De acuerdo con datos de la Reserva Federal de Estados Unidos, la tasa de interés de los títulos del tesoro, ajustada a la inflación, pasó de 1.27\% en 2003, a 1.04\% en 2004. 
La relación entre el DCEU y los cambios en las cuentas corrientes nacionales

La política estratégica de Estados Unidos, dirigida a financiar su crecimiento económico mediante ahorros foráneos, depende de la condición superavitaria de la cuenta corriente que tienen las naciones prestamistas, como han sido Japón y Alemania tradicionalmente, y hoy en día, China. En 2007 China se posicionó como el principal exportador neto de capital, el 21.3\% de los flujos financieros externos corrió por su cuenta, Alemania siguió con el $14.5 \%$ y Japón con el 12.1\%. La participación de los restantes diez primeros exportadores de capital, todos países en desarrollo y la mayoría del Este de Asia, fue menor a dos dígitos. Mientras, Estados Unidos continuó siendo el principal importador neto de capital, al recibir el $49.2 \%$ de los flujos financieros externos en 2007, seguido por España (9.8\%) y el Reino Unido (8.0\%) (FMI, World Economic Outlook, 2010).

Paralelamente al cúmulo de superávit externos, la región asiática ha mantenido un creciente volumen de reservas internacionales. Japón y China han logrado adquirir, a

La política estratégica de Estados Unidos, dirigida a financiar su crecimiento económico mediante ahorros foráneos, depende de la condición superavitaria de la cuenta corriente que tienen las naciones prestamistas, como han sido Japón y Alemania tradicionalmente, y hoy en día, China través de sus reservas internacionales, una significativa cantidad de valores estadounidenses. Durante el segundo semestre del 2002 y el primer semestre del 2004, Japón destinó casi el monto total de sus reservas a la compra de títulos de Tesoro de Estados Unidos. En 2003 China consignó aproximadamente el $75 \%$ de sus reservas a la adquisición de valores estadounidenses y poco menos del $50 \%$ a Títulos del Tesoro de Estados Unidos (Global Financial Stability Report, 2006:35). Aunque en abril de 2008, China sobrepasó a Japón como el primer comprador de títulos del Tesoro de Estados Unidos, Japón continúa con esta primacía, y en diciembre de 2008 logró una máxima participación de $8.38 \%$ (gráfica 2). 


\section{Gráfica 2}

Participación de China y Japón sobre las letras

del Tesoro de Estados Unidos

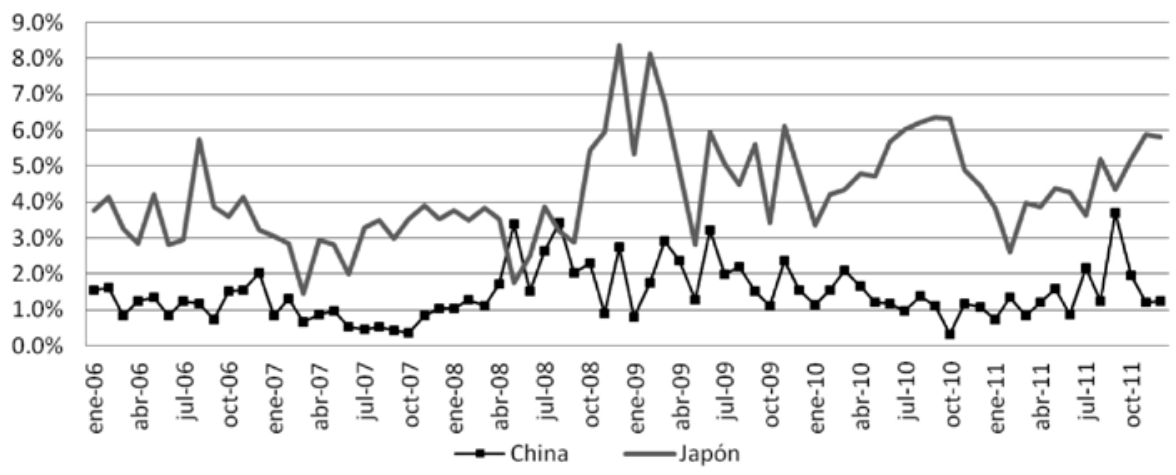

Fuente: U.S. Department of the Treasury: www.treasury,gov/resource-center/data-chartcenter/tic/Pages/ticsec.aspx, consultado febrero 20, 2012.

La sostenibilidad del déficit comercial de Estados Unidos

En la medida en que el financiamiento del déficit comercial depende de la inversión extranjera, una de las tesis más comentadas (Griswold, 2001; Pakko, 1999) es la vulnerabilidad sistémica ocasionada por una posible salida masiva de capitales; un escenario de "duro aterrizaje" producto de una significativa devaluación del dólar y recesión de la economía estadounidense, que resultaría en un círculo vicioso de caída del ingreso, consumo y valor del dólar. Sin embargo, también existe la hipótesis de un "suave aterrizaje" si el ciclo de negocios, la tasa de interés, los tipos de cambio y los ingresos relativos nacionales se ajustan "automáticamente" para restablecer un comercio más equilibrado con los socios comerciales estadounidenses.

En el corto plazo, el problema de la sostenibilidad del DCEU está principalmente relacionado con la composición de la cuenta de capital encargada de financiarlo. Esta no se encuentra integrada mayoritariamente por inversión directa (asociada con nuevos bienes de capital) sino por inversión de portafolio, la cual tiene un grado de riesgo mayor debido a las características del desregulado sector financiero internacional. 


\section{El déficit comercial de Estados Unidos en cifras}

El DCEU está concentrado en manufacturas y combustibles minerales y procede mayoritariamente de las relaciones comerciales con el Este de Asia. Sectorialmente, de acuerdo con la clasificación de la industria norteamericana (NAICS), entre 2000 y 2007 la rama con un mayor saldo comercial negativo fue el de computadoras y productos electrónicos, sector que ascendió su participación en el déficit manufacturero estadounidense de $22.54 \%$ en 2000 a $26.14 \%$ en 2007, seguido por equipo eléctrico, aplicaciones y componentes, el cual detentó el 3.58\% del déficit manufacturero en 2000 y el $4.99 \%$ en 2007. Contrariamente, durante estos siete años, la contribución del sector de equipo de transporte se desplazó del $23.1 \%$ del déficit manufacturero al $11.22 \%$, y el ramo de ropa y accesorios descendió ligeramente de $13.85 \%$ a $11.24 \%$. Paralelamente, entre 2000 y 2007, las exportaciones estadounidenses de alta tecnología descendieron de $24.66 \%$ a $10.89 \%$. No obstante, este tipo de exportaciones aumentaron hacia China de $2.07 \%$ a $6.75 \%$, respecto al total de exportaciones estadounidenses de alta tecnología (U.S. Census Bureau, 2009:787,796).

Regionalmente, el DCEU procede mayoritariamente de las relaciones comerciales con el Este de Asia. En 1990 el 76.56\% del déficit estadounidense correspondió a esta región y tal proporción fue aproximadamente $50 \%$ en 2007. La Unión Europea también ha ganado espacio sobre el DCEU. En 1990 Estados Unidos tuvo un superávit de $4.28 \%$ con la Unión Europea, y diecisiete años después, esta área fue responsable del $12.84 \%$ del DCEU.

En términos nacionales, la composición del DCEU ha cambiado significativamente. Japón participó con el 58.7\% del DCEU en 1992 y con el 27.67\% en 1995, seguido por Canadá: 9.78\%, y México: 7.23\%. En 2005 China tomó el primer lugar, mientras otras economías del Este de Asia de ingreso alto y medio han seguido la trayectoria decreciente de Japón sobre la participación en el DCEU (United Nations Commodity Trade, 2010) (gráfica 3). De forma tal que el desplazamiento de un número específico de economías asiáticas del mercado estadounidense por parte de China está entre los hechos más sobresalientes en el estudio del DCEU (gráfica 4). 


\section{Gráfica 3}

Déficit comercial de Estados Unidos

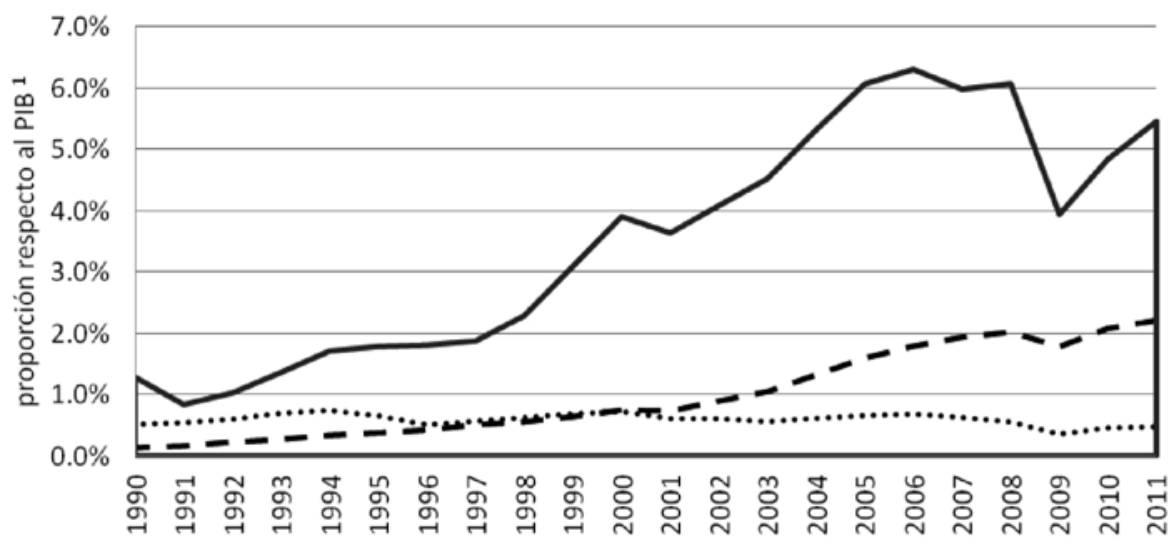

::Def. EE.UU con Japón

CDef. China con EE.UU

口Def. bienes de EE.UU

${ }^{1}$ PIB ajustado al año 2005

Fuente: para PIB: Bureau of Economic Analysis. www.bea.gov/index.htm; para comercio de Japón y China en: Cepal, Magic Plus. www.eclac.org/magic/demo.aspx?id=0, consultado julio 15, 2012.

\section{Gráfica 4}

Participación de China en el déficit comercial de Estados Unidos

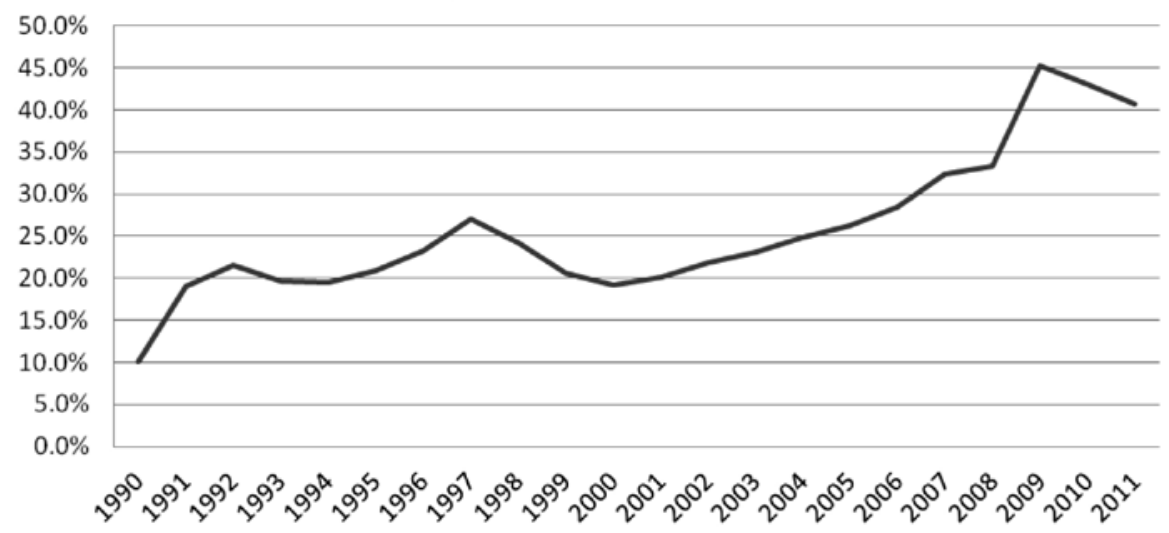

Fuente: Cepal, Magic Plus: www.eclac.org/magic/demo.aspx?id=0, consultado julio 15, 2012. 


\section{La visión estructural del déficit comercial de Estados Unidos}

De acuerdo con el enfoque estructural del déficit comercial, la productividad laboral, las barreras comerciales, y la calidad del producto e innovación tecnológica son elementos que determinan los parámetros bajo los cuales operan las principales políticas macroeconómicas (fiscal, monetaria y tipo de cambio), y estas políticas fijan el grado en que los factores microeconómicos se manifiestan en los balances de las transacciones internacionales de un país. En este sentido, la visión heterodoxa comparte con el enfoque clásico que la balanza comercial estadounidense es una variable macroeconómica afectada, en principio, por los ahorros nacionales. Sin embargo, el pensamiento no convencional enfatiza que los factores estructurales intervienen en el DC.

Las causas estructurales más importantes del DCEU se relacionan con la fragmentación e internacionalización de los procesos de producción dirigidos por las empresas transnacionales (ETN), que han resultado en un elevado comercio intra-industrial (transacciones de partes y componentes de un mismo bien final) y altos coeficientes de importación. Además, están presentes las políticas comerciales con fuertes barreras no arancelarias y las políticas industriales asistidas por el Estado ${ }^{8}$ que han seguido Japón, los países del denominado "milagro asiático" (Hong Kong, Taiwán, Corea y Singapur), Malasia, Tailandia, Indonesia y China, bajo el argumento de "industrias nacientes".

Otra fuente estructural del DCEU son los factores subyacentes al crecimiento económico de China, los cuales pueden ser clasificados en exógenos y endógenos. Entre los primeros elementos, este artículo destaca el desplazamiento de las capacidades productivas estratégicas del Este de Asia hacia China y la presencia en China de filiales de reconocidas ETN.

Los factores endógenos del crecimiento de China no son abordados en este texto. Sin embargo, cabe mencionar, que la política económica de metas numéricas de desempeño establecidas en los Planes Quinquenales de Desarrollo Económico y Social, el modelo de desarrollo de infraestructura pública y propiedad estatal sobre sectores estratégicos, y la oferta de mano de obra de bajo costo (altamente disciplinada y cada día más capacitada), se encuentran

8. En general, se trata de un conjunto de políticas estratégicas dirigidas a seleccionar un grupo de industrias con un alto valor agregado por trabajador y una significativa difusión tecnológica hacia el resto de la economía nacional. Estas industrias reciben un conjunto de apoyos gubernamentales de control de importaciones y promoción de exportaciones, junto con incentivos en actividades de investigación y desarrollo. 
entre los elementos domésticos más importantes del crecimiento económico de China, y por ende, de su significativa participación en el DCEU.

\section{La plataforma exportadora del Este de Asia operando en China}

El traslado deliberado de particulares capacidades productivas hacia China se inició en la década de los ochenta del siglo xx, cuando Japón detonó un firme proceso de reconversión industrial en medio de una política sectorial asistida por el gobierno. Esta iniciativa comenzó con la subcontratación en países de bajos salarios, particularmente del Este de Asia, de actividades manufactureras intensivas en fuerza de trabajo y tecnológicamente estandarizadas. De forma tal que Japón creó un "sistema transfronterizo de sub-contratación", incorporando una gran "masa de fuerza de trabajo permisiva y trabajadora" capaz de detonar el desarrollo del "archipiélago capitalista del Este asiático" (Arrighi, 2010: 336-370). El primer grupo de países que se benefició de este proyecto fueron las nuevas economías industrializadas del Este de Asia (NEIS): Hong Kong, Singapur, Corea y Taiwán; después, cuatro miembros de la Asociación de Naciones del Sureste Asiático (ASEAN-4): Indonesia, Filipinas, Tailandia y Malasia; y recientemente, China y Vietnam.

De acuerdo con Arrighi (Op. Cit.), el proceso anterior está relacionado con el desarrollo industrial en oleadas del Este de Asia. Primero, las NEIS avanzaron y su ventaja comparativa en la producción industrial de menor valor agregado descendió. El resultado fue la búsqueda de otras áreas geográficas con bajos costos laborales como la ASEAN-4, un conjunto de economías donde el capital procedente de Japón y las primeras nuevas economías industrializadas del Este de Asia comenzaron a invertir. La ASEAN-4 repitió el curso de las NEIS, y trasladó las fases productivas de bajo valor agregado hacia China y Vietnam, lo que dio lugar a un "régimen de acumulación emergente" sostenido en el "reciclaje de la ventaja comparativa".

El proceso anterior, identificado con la figura de flying geese, representa la evolución sistémica del Este de Asia a partir de la oferta de recursos, la fuerza de trabajo y el capital monetario. Este proceso ha permitido la integración de espacios productivos heterogéneos y el posicionamiento de China como un elemento clave en la interdependencia del Este de Asia, que resulta de su función en los sistemas de producción compartida intra-regionales, o su participación en las cadenas de producción, cuyo factor subyacente son las 
significativas diferencias salariales entre los países orientales (Athukorala, 2010:409).

Aproximadamente, el costo laboral promedio (por hora) en la industria manufacturera china es del $6 \%$ respecto a Japón y 20\% en relación con Taiwán, Corea, Hong Kong y Singapur (Ping, 2010:77). China, junto con los países de la ASEAN (excepto Singapur), mantiene los salarios más precarios, incluso por debajo, o comparables, a aquellos de la periferia europea y México (Athukorala, Op Cit. 409). Aunque, cabe mencionar, hoy en día, existen "locaciones hambrientas", que son economías con una gran cantidad de mano de obra de bajo costo, dispuestas a hacer concesiones en materia laboral a cambio de inversión extranjera.

De acuerdo con datos de Bradsher (2008), los trabajadores no calificados en las provincias costeras de China (con acceso a importantes puertos) ahora ganan $\$ 120$ dólares mensuales por una jornada de trabajo de 40 horas a la semana, esto es, menos de un dólar por hora. Al mismo tiempo, los trabajadores en Vietnam ganan menos de 50 dólares al mes por una jornada de 48 horas de trabajo de lunes a sábado. Esta situación explica la estrategia corporativa denominada "China más uno", la cual busca extender la base productiva asiática, particularmente hacia Vietnam, con el objetivo de mitigar los riesgos de la sobre-dependencia respecto a las empresas de un solo país. Por ejemplo, en 2008, la firma de textiles Hanesbrands construyó una empresa automatizada en Nanjing (China) y otra en Vietnam, además compró dos empresas más en Tailandia con el objetivo de reducir $\operatorname{costos}^{9}$.

Los procesos referidos en párrafos anteriores están materializados en una plataforma exportadora regional dentro de China, líder en la rama eléctrica y electrónica, junto con la textil y otras ramas manufactureras intensivas en mano de obra, que opera mayoritariamente a través de inversión extranjera directa (IED) y empresas de inversión extranjera (EIE). Respecto a la IED en China, durante la década de los noventa, se advirtió el ascenso constante de la participación de Estados Unidos y la Unión Europea, y el descenso de Hong Kong, Taiwán y Japón en el nuevo milenio. No obstante, las economías asiáticas continúan detentando poco más del 70\% de la IED en China debido

9. En 2008 Hanesbrands anunció el cierre de nueve plantas entre El Salvador, Costa Rica, Hondura y Estados Unidos, afectando a 8,100 empleados. Paralelamente, esta firma notificó un aumento de sus operaciones en Asia y América Central (cnnexpansión.com, 2008). 
al ingreso de las economías de la Asociación de Naciones del Sureste Asiático en la dinámica económica de China, sobre todo, Singapur, Macao, y Corea.

En 1999, Estados Unidos alcanzó el 11.96\% de los proyectos extranjeros chinos y un año después sumó el $10.77 \%$ de la IED realizada en el país asiático. Sin embargo, en 2007, la economía estadounidense solo fue responsable del $6.80 \%$ del número de proyectos extranjeros y del 3.13\% de la inversión extranjera realizada en China. Mientras, la Unión Europea no ha logrado superar su máxima participación sobre el valor de la IED realizada en China de 11.11\% (1999), y solo alcanzó el 4.60\% en 2007 después de una caída de $5.1 \%$ (Ibídem.). En promedio, el capital de las EIE operando en China procede de Estados Unidos, Hong Kong, la Unión Europea, Japón y Taiwán. Estas cinco economías contribuyeron con el $88 \%$ de las exportaciones chinas realizadas por las EIE en 1993 y el $75.48 \%$ en 2007 (Foreign Trade Department of the Ministry of Commerce, MOFCOM, 2009).

En el transcurso de la apertura económica, las exportaciones chinas elaboradas por las EIE han marcado una creciente dependencia de las importaciones. En el periodo 2003-2007, las EIE realizaron el 57.61\% del comercio chino y el $39.15 \%$ de este fue por procesamiento comercial de insumos importados (Chen; et al., 2007:12-17).

Los principales commodities exportados por las EIE coinciden con los commodities importados. En el transcurso de 2004-2007, la participación promedio del rubro "productos mecánicos y eléctricos" sobre los commodities chinos exportados fue de $70.55 \%$. Dentro de este sector, la categoría "productos nuevos y de alta tecnología" sumó el $38.16 \%$, "equipo de procesamiento de datos automáticos y componentes": $15.09 \%$ y “circuitos integrados y paquete microelectrónico": $3.07 \%$. Respecto a los commodities chinos importados, la contribución promedio del rubro "productos mecánicos y eléctricos" fue de $68.64 \%$, para "productos nuevos y de alta tecnología": 39.05\%, "circuitos integrados y paquetes micro electrónicos": $18.48 \%$, y "equipo de procesamiento de datos automáticos y componentes": 3.08\% (MOFCOM, 2009). Este patrón de exportaciones e importaciones de las EIE explica la venta foránea de mercancías chinas de alto contenido tecnológico.

El comercio de China en bienes de alta tecnología

De acuerdo con el National Center for Science and Engineering Statistics (NCSES, 2010), Estados Unidos realizó el 14\% de las ventas mundiales de las 
mercancías de alta tecnología en 2008, siete puntos porcentuales menos respecto a 1995; mientras la proporción de China aumentó de 6\% en 1995 a 20\% en 2008. En 2001 la posición comercial de China en este tipo de productos transitó de un equilibrio a un superávit, el cual aumentó de forma acelerada de \$13 mil millones de dólares en 2003, a \$130 mil millones de dólares en 2008, en respuesta al desempeño de la rama: "información y tecnología de la comunicación".

En general, la región asiática ha logrado mantener una mayor participación en el comercio de alta tecnología. Durante la pasada década, el superávit comercial de Asia-9 logró pasar de menos de $\$ 50$ mil millones de dólares (1995) a más de $\$ 220$ mil millones de dólares (2008), como resultado del significativo superávit en los bienes de "tecnología de la información" (TI).

El crecimiento de las exportaciones de alta tecnología en China ha sido un proceso paralelo al crecimiento de sus importaciones procedentes de Japón, Corea y Taiwán. En 1990 el 7.3\% de las exportaciones japonesas de alta tecnología tuvieron a China como destino. En 2008 esta proporción alcanzó el $28.5 \%$ debido a una tendencia ascendente más pronunciada entre 2001 y 2003. El mismo comportamiento presentaron Corea y Taiwán, economías que realizaron en China el $5.6 \%$ y $7.7 \%$ de sus ventas foráneas de alta tecnología en 1990, y el $36.9 \%$ y $50.4 \%$ en 2008 (Ibídem.), respectivamente.

En el transcurso del siglo xxi, China, Malasia, México, Japón, Singapur y Taiwán, han sido las economías responsables de poco más de 3/4 de las importaciones estadounidenses de "computadoras y productos electrónicos". En 2000 $19.55 \%$ y $12.05 \%$ de las importaciones estadounidenses de "computadoras y productos electrónicos" procedieron de Japón y China, respectivamente. En 2009 éstas proporciones fueron $2.95 \%$ y $61.42 \%$, lo cual colocó a Japón en el quinto lugar y a China en el primer escaño de los proveedores estadounidenses en la rama. Durante estos años, también las participaciones de Singapur y Taiwán descendieron estrepitosamente, mientras las de México y Malasia se mantuvieron estables.

Sin embargo, existe una dimensión real de la dinámica exportadora china en bienes de alta tecnología que dos hechos ayudan a ejemplificar. Primero, al mismo tiempo que China ha desplazado a Japón como el primer proveedor de "computadoras y productos electrónicos" hacia Estados Unidos, la IED estadounidense en China ha aumentado significativamente en el sector. Entre 2000 y 2008 , el $8.51 \%$ y $12.9 \%$ de la IED estadounidense en China fue destinada al citado rubro de mercancías. En cambio, estas proporciones para 
Japón fueron de 6\% y 4.31\%, respectivamente (BEA, International Economic Accounts, 2010).

Segundo, de acuerdo con cifras del 2010, el 32.4\% del valor agregado del Apple Ipod, uno de los bienes de consumo de tecnología más avanzados, procede de Estados Unidos por las actividades de diseño, marketing y manufactura de componentes (en 2005, tal proporción fue de 38.7\%). El resto del valor agregado de este famoso producto está dividido entre Corea, Japón y Taiwán por la manufactura de componentes, mientras China por el ensamble final solo aporta el $1.6 \%$ del valor agregado, $0.2 \%$ menos respecto al 2005 . El ascenso significativo de Corea ha destacado porque en cinco años su participación en el valor agregado del Apple Ipod aumentó 6.4\% (Gráfica 5). Por lo tanto, el soporte fundamental de las exportaciones chinas de alta tecnología son las EIE produciendo con altos índices de especialización vertical (valor de bienes intermedios importados para fabricar una unidad de producto en el sector doméstico), particularmente se trata de las filiales de transnacionales de capital estadounidense.

Ventaja comparativa revelada ${ }^{10}$ en el Este de Asia, un comparativo entre China-Estados Unidos

Una lectura más clara del patrón comercial del Este de Asia y China se obtiene a través del índice de ventaja comparativa revelada (IVCR), el cual considera tanto la dinámica de exportación como los niveles de importación. En general, la referida región no posee una ventaja comparativa revelada (VCR) en el sector primario. Únicamente Indonesia y Malasia tienen una VCR positiva en

10. De acuerdo con Vollraht (1991), la expresión matemática del índice de la ventaja comparativa revelada es:

VCR: ventaja comparativa revelada

VCE: ventaja comparativa revelada de exportaciones

VCI: ventaja comparativa revelada de importaciones

i: país en estudio

$r$ : el resto del mundo que interviene en el intercambio comercial de dos bienes

a: bien en estudio

n: resto de bienes

$V C R_{a}^{i}=V C E_{a}^{i}-V C M_{a}^{i}$

$V C E_{a}^{i}=\operatorname{In}\left[\left(X_{a}^{i} / X_{n}^{i}\right) /\left(X_{a}^{r} / X_{n}^{r}\right)\right]$

$V C M_{a}^{i}=\ln \left[\left(M_{a}^{i} / M_{n}^{i}\right) /\left(M_{a}^{r} / M_{n}^{r}\right)\right]$ 


\section{Gráfica 5}

Composición de la cadena de valor del Appli Ipod por locación, 2010

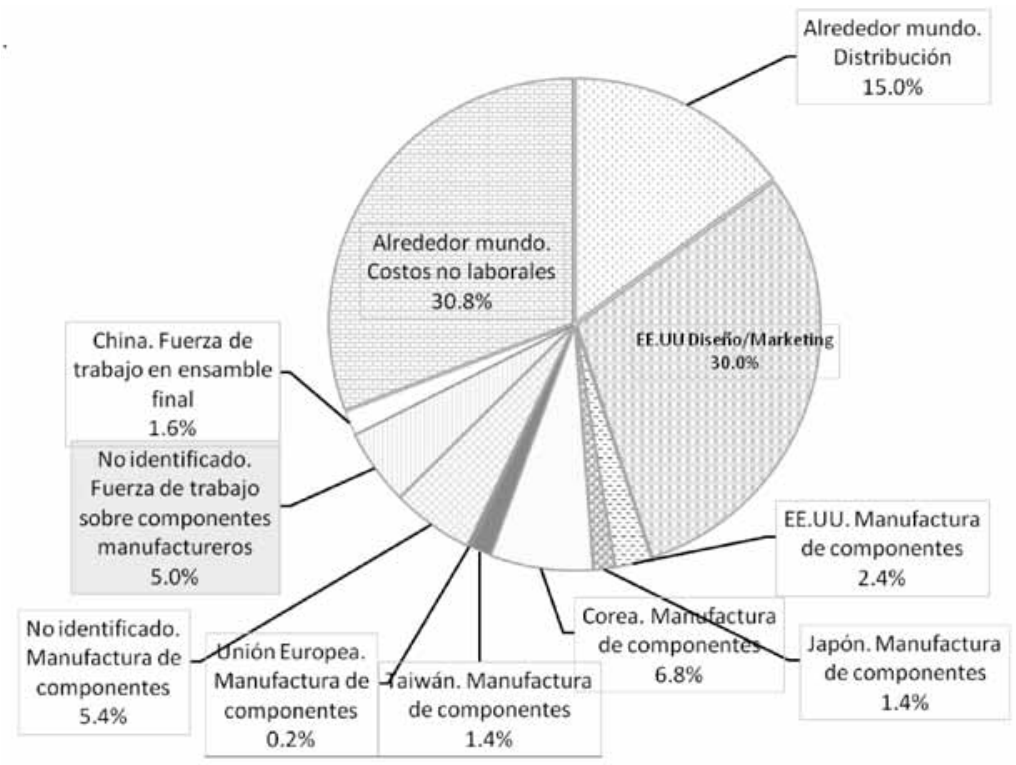

Fuente: National Center for Science and Engineering Statistics, "Industry, Technology, and the Global Marketplace", Science and Engineering Indicators 2012, National Science Foundation, Arlington, VA, enero de 2012, p.30.

los "productos primarios" (PP) y "manufacturas basadas en recursos agrícolas" (M1). La razón de este desempeño es que ambos países revelan ventajas comparativas para abastecer su mercado interno y logran concentrar sus exportaciones en estos sectores. En el caso de Malasia, también influye que el país no interviene en forma significativa en el comercio mundial de PP y su consumo doméstico es limitado (Cuadros 1.1 y 1.2).

En 1995, China y Tailandia perdieron la VCR sobre PP, y Tailandia comenzó a concentrar su ventaja en M1, sector donde China ha logrado índices positivos a partir del segundo quinquenio del siglo XxI. En cambio, las "manufacturas basadas en otros recursos agrícolas" (M2) ha sido un sector con un bajo desempeño en la región, únicamente Singapur mantuvo una ventaja comparativa revelada positiva hasta 2005 (Cuadro 1.3). Respecto a las manufacturas intensivas en mano de obra: "textiles, prendas de vestir y calzado" (MBT1) y 
"otras manufacturas de baja tecnología" (MBT2), Corea, China y Hong Kong presentan una clara especialización productiva en estos sectores desde 1995. Entre estas tres economías sobresale el valor de los índices de China, los cuales superan los de Corea y Hong Kong (Cuadro 1.4).

Dentro de las manufacturas básicas, Indonesia, Filipinas, Malasia y Tailandia mantienen una ventaja comparativa revelada en MBT1 con índices significativos entre 1 y 3 , porque estas economías tienen una demanda doméstica limitada en estos productos, revelan una ventaja comparativa para abastecer su mercado interno y logran concentrarse en las exportaciones de estos bienes. Después del 2000, Indonesia perdió la ventaja en MBT2, mientras Malasia la alcanzó entre 2000 y 2005, y Japón detentó una ventaja en este sector hasta 2005 (Cuadro 1.5).

En relación a las manufacturas medias: "productos automotrices de mediana tecnología" (MMT1), "productos químicos y metales básicos de mediana tecnología" (MMT2) y "productos de ingeniería de mediana tecnología" (MMT3), el liderazgo en la región lo mantiene Japón, porque el país del sol naciente cuenta con una dinámica positiva de exportación en MMT1 y ha construido una alta integración comercial con el área oriental, particularmente con China, en bienes intermedios. El otro país con un significativo desempeño en estos rubros es Corea, específicamente en MMT1. En los primeros años del nuevo milenio, Hong Kong ha comenzado un desempeño positivo, primero en el rubro MT3 y después en MT2 (Cuadros 1.6 - 1.8).

Respecto a las manufacturas de alta tecnología: "productos electrónicos y eléctricos de alta tecnología" (MAT1) y "otros productos de alta tecnología" (MAT2), Japón, Corea y Tailandia se distinguen por sus continuos índices de ventaja comparativa revelada positivos en la región. Aunque, en el caso de Japón, estos valores no son significativamente mayores a 1, pero sí mayores a los de Corea y Tailandia. En los tres casos los índices revelan una clara ventaja comparativa de exportación dentro del área, lo cual corrobora la triangulación de los procesos productivos en el Este de Asia.

Entre 1995 y 2005, el IVCR de Filipinas en MAT1 comenzó a crecer y logró un máximo de 0.96 en el año 2000. También ascendió el índice de Malasia, pero a un ritmo menor, incluso descendió significativamente en 2005; mientras China, únicamente en ese año, alcanzó un índice positivo de 0.17 en el sector MAT1 (Cuadro 1.9). Por último, el Este de Asia no tiene un desempeño significativo en el sector de MAT2. Hong Kong y Japón lograron índices positivos entre 2000 y 2009 , pero los valores fueron significativamente 
bajos. En el caso de Japón de 0.08 y 0.07, y para Hong Kong de 0.09 y 0.13, respectivamente (Cuadro 1.10 ).

El comportamiento de los indicadores antes analizados ayuda a sostener la tesis de que China es una plataforma regional de exportación distante de lograr un patrón comercial sostenido en una ventaja comparativa dentro de sectores de alta tecnología. Asimismo, se observa que, dentro de la región, Japón continúa destacándose por una plataforma comercial intensiva en conocimiento y alta tecnología. El resto de las economías asiáticas, específicamente Corea, Hong Kong, Tailandia, Singapur, Filipinas y Malasia, sostienen una importante ventaja comparativa revelada en MAT1, lo que apoya la dinámica regional de crecimiento económico en torno al sector de "electrónicos y tecnología".

Finalmente, un comparativo entre China y Estados Unidos de los índices de ventaja comparativa revelada apoya la hipótesis de que, específicamente, los sectores "desplazados" por China han sido los intensivos en mano de obra y el primer rubro de manufacturas medias. Estados Unidos mantiene un conjunto mayoritario de índices positivos a partir del sector MMT2 y demuestra ser una economía concentrada en la exportación de alta tecnología (Cuadro 1.A). Sin embargo, Japón tiene una mayor ventaja competitiva respecto a Estados Unidos en cinco sectores de mediana y alta tecnología, que integran el comercio manufacturero a escala mundial. Estos son: productos automotrices, productos químicos y metales básicos, productos de ingeniería, productos eléctricos y electrónicos de alta tecnología, y otros productos de alta tecnología.

\section{Cuadro 1.A}

Índice de ventaja comparativa revelada en Estados Unidos

\begin{tabular}{lrrrr}
\hline & 1995 & 2000 & 2005 & 2009 \\
\hline Productos primarios & 0.05 & -0.68 & -0.88 & -0.64 \\
Manufacturas basadas en recursos agrícolas & 0.22 & 0.14 & 0.02 & 0.17 \\
Manufacturas basadas en otros recursos agrícolas & 0.19 & -0.17 & -0.04 & 0.28 \\
Textiles, prendas de vestir y calzado & -1.46 & -1.38 & -1.50 & -1.81 \\
Otras manufacturas de baja tecnología & -0.32 & -0.22 & -0.23 & -0.22 \\
Productos automotrices de mediana tecnología & -0.70 & -0.80 & -0.46 & -0.27 \\
Productos químicos y metales básicos de mediana & 0.81 & 0.90 & 0.91 & 1.03 \\
tecnología & & & & \\
Productos de ingeniería de mediana tecnología & 0.15 & 0.41 & 0.45 & 0.26 \\
Productos electrónicos y eléctricos de alta tecnología & -0.02 & 0.29 & 0.19 & -0.27 \\
\hline
\end{tabular}

Fuente: United Nations Commodity Trade Statistics Database: http://comtrade.un.org/db/, fecha de consulta octubre 2010. 
El déficit comercial de Estados Unidos frente a la entrada de China a su mercado

Cuadro 1

Índice de ventaja comparativa revelada en economías de Asia

\begin{tabular}{|c|c|c|c|c|c|c|c|c|}
\hline & \multicolumn{4}{|c|}{ 1.1. Productos primarios } & \multicolumn{4}{|c|}{$\begin{array}{l}\text { 1.2. Manufacturas basadas en } \\
\text { recursos agrícolas }\end{array}$} \\
\hline & 1995 & 2000 & 2005 & 2009 & 1995 & 2000 & 2005 & 2009 \\
\hline Corea & -2.16 & -2.80 & -2.88 & -2.42 & -0.68 & -0.39 & -0.31 & -0.36 \\
\hline China & 0.09 & -0.74 & -1.22 & -0.28 & -0.17 & -0.40 & -0.13 & 0.07 \\
\hline Finlandia & -0.42 & -1.59 & -1.26 & -0.39 & 0.55 & -0.16 & 0.30 & 0.40 \\
\hline Hong Kong & -0.53 & -0.67 & -0.64 & -0.12 & -0.34 & -0.46 & -0.70 & -0.11 \\
\hline Indonesia & 1.33 & 0.64 & 0.93 & 1.46 & 1.31 & 0.76 & 1.10 & 1.16 \\
\hline Japón & -3.57 & -3.66 & -2.17 & -3.47 & -1.96 & -1.63 & -1.32 & -1.59 \\
\hline Malasia & 0.73 & 0.39 & 0.50 & 0.18 & 1.48 & 0.96 & 0.94 & 0.81 \\
\hline Singapur & -0.72 & -1.46 & -1.67 & -0.68 & -0.24 & -0.38 & -0.19 & -0.05 \\
\hline \multirow[t]{3}{*}{ Tailandia } & 0.65 & -0.40 & -0.69 & -0.09 & 0.80 & 1.07 & 1.13 & 0.84 \\
\hline & \multicolumn{4}{|c|}{$\begin{array}{l}\text { 1.3. Manufacturas basadas en otros } \\
\text { recursos agrícolas }\end{array}$} & \multicolumn{4}{|c|}{$\begin{array}{l}\text { 1.4. Textiles, prendas de vestiry } \\
\text { calzado }\end{array}$} \\
\hline & 1995 & 2000 & 2005 & 2009 & 1995 & 2000 & 2005 & 2009 \\
\hline Corea & -0.87 & -0.13 & -0.30 & -0.86 & 1.15 & 1.16 & 0.35 & 1.16 \\
\hline China & -0.08 & -0.52 & -0.87 & -0.65 & 1.72 & 1.85 & 2.22 & 2.90 \\
\hline Finlandia & -0.66 & -0.71 & -0.78 & -1.26 & 1.01 & 1.01 & 1.02 & 1.31 \\
\hline Hong Kong & -0.39 & -0.69 & -0.85 & -0.34 & 0.44 & 0.38 & 0.27 & 0.84 \\
\hline Indonesia & -0.39 & -1.00 & -1.18 & -0.54 & 1.52 & 1.44 & 2.01 & 1.35 \\
\hline Japón & -0.83 & -0.83 & -0.74 & -0.71 & -2.23 & -2.11 & -2.12 & -2.41 \\
\hline Malasia & -0.79 & -0.68 & -0.64 & -0.82 & 0.79 & 0.84 & 0.79 & 0.91 \\
\hline Singapur & 0.67 & 0.30 & 0.75 & -0.43 & -0.32 & -0.25 & -0.22 & 0.08 \\
\hline \multirow[t]{3}{*}{ Tailandia } & -0.59 & -0.18 & 0.00 & 0.06 & 2.21 & 1.41 & 1.28 & 1.92 \\
\hline & \multicolumn{4}{|c|}{$\begin{array}{l}\text { 1.5. Otras manufacturas de baja } \\
\text { tecnología }\end{array}$} & \multicolumn{4}{|c|}{$\begin{array}{l}\text { 1.6. Productos automotrices de } \\
\text { mediana tecnología }\end{array}$} \\
\hline & 1995 & 2000 & 2005 & 2009 & 1995 & 2000 & 2005 & 2009 \\
\hline Corea & 0.54 & 0.54 & 0.13 & 0.19 & 1.72 & 2.31 & 2.28 & 1.28 \\
\hline China & 3.84 & 3.71 & 3.19 & 4.43 & -0.81 & -0.07 & -0.03 & -1.41 \\
\hline Finlandia & -0.06 & -0.44 & -0.43 & 0.09 & -1.44 & -0.59 & 0.36 & -1.54 \\
\hline Hong Kong & 0.40 & 0.38 & 0.24 & 0.52 & -1.02 & -0.81 & -0.48 & -0.03 \\
\hline Indonesia & 0.03 & 0.24 & -0.12 & -0.14 & -2.47 & -2.03 & -1.34 & -2.09 \\
\hline Japón & 0.06 & 0.06 & 0.14 & -0.07 & 1.84 & 2.24 & 2.36 & 2.21 \\
\hline Malasia & -0.36 & -0.18 & 0.05 & -0.33 & -1.98 & -1.66 & -1.64 & -2.12 \\
\hline Singapur & -0.54 & -0.20 & 0.03 & -0.27 & -0.83 & -1.13 & -0.38 & -0.83 \\
\hline Tailandia & -0.15 & -0.14 & -0.20 & -0.18 & -2.00 & 0.11 & 0.81 & -1.41 \\
\hline
\end{tabular}


Ximena Valentina Echenique Romero

\begin{tabular}{|c|c|c|c|c|c|c|c|c|}
\hline & \multicolumn{4}{|c|}{$\begin{array}{l}\text { 1.7. Productos químicos y metales } \\
\text { básicos de mediana tecnología }\end{array}$} & \multicolumn{4}{|c|}{$\begin{array}{l}\text { 1.8. Productos de ingeniería de } \\
\text { mediana tecnología }\end{array}$} \\
\hline & 1995 & 2000 & 2005 & 2009 & 1995 & 2000 & 2005 & 2009 \\
\hline Corea & 0.45 & 0.30 & 0.11 & 0.46 & -0.39 & 0.16 & 0.27 & -0.08 \\
\hline China & -0.95 & -1.02 & -0.72 & -0.52 & -1.06 & -0.26 & -0.04 & -0.45 \\
\hline Finlandia & -1.51 & -2.14 & -1.63 & -1.19 & -1.18 & -0.28 & -0.22 & -0.70 \\
\hline Hong Kong & -0.26 & -0.22 & -0.23 & 0.34 & -0.01 & 0.04 & 0.05 & 0.02 \\
\hline Indonesia & -0.18 & -0.85 & -0.82 & -0.06 & -1.36 & -1.40 & -1.06 & -1.12 \\
\hline Japón & 0.61 & 0.67 & 0.82 & 0.52 & 1.62 & 1.39 & 1.17 & 1.12 \\
\hline Malasia & -0.58 & -0.40 & -0.22 & -0.95 & -0.47 & -0.43 & -0.23 & -0.17 \\
\hline Singapur & -0.32 & -0.01 & 0.48 & -0.12 & -0.34 & -0.40 & -0.02 & -0.24 \\
\hline \multirow[t]{3}{*}{ Tailandia } & -0.63 & -0.59 & -0.39 & -0.76 & -0.95 & -0.39 & -0.15 & -0.68 \\
\hline & \multicolumn{4}{|c|}{$\begin{array}{l}\text { 1.9. Productos electrónicos y } \\
\text { eléctricos de alta tecnología }\end{array}$} & \multicolumn{4}{|c|}{$\begin{array}{l}\text { 1.10. Otros productos de alta } \\
\text { tecnología }\end{array}$} \\
\hline & 1995 & 2000 & 2005 & 2009 & 1995 & 2000 & 2005 & 2009 \\
\hline Corea & 0.89 & 0.53 & 0.79 & 0.82 & -1.81 & -1.53 & -0.35 & -0.82 \\
\hline China & -0.35 & -0.27 & 0.17 & -1.11 & -0.47 & -0.51 & -1.21 & -0.86 \\
\hline Finlandia & 0.28 & 0.96 & 0.73 & -1.06 & -2.56 & -1.32 & -0.55 & -1.91 \\
\hline Hong Kong & -0.12 & -0.17 & 0.02 & -1.19 & -0.39 & 0.09 & -0.10 & 0.13 \\
\hline Indonesia & -0.07 & 1.13 & 0.45 & -0.57 & -1.31 & -1.58 & -1.73 & -1.42 \\
\hline Japón & 1.05 & 0.52 & 0.40 & 1.05 & -0.21 & 0.08 & -0.03 & 0.07 \\
\hline Malasia & 0.21 & 0.24 & 0.06 & 0.20 & -0.88 & -0.81 & -0.61 & -0.09 \\
\hline Singapur & 0.52 & 0.50 & 0.64 & 0.93 & -0.80 & -0.56 & -0.21 & -0.71 \\
\hline Tailandia & 0.24 & 0.16 & 0.28 & 0.23 & -0.93 & -1.50 & -0.71 & -0.35 \\
\hline \multicolumn{9}{|c|}{$\begin{array}{l}\text { 2/ Índices entre } 1 \text { y } 2 \text { indican la presencia de industrias exportadoras competitivas e índices } \\
\text { mayores a } 2 \text { muestran una concentración en exportaciones en el ramo por un suficiente } \\
\text { abasto nacional. Índices negativos manifiestan una falta de competitividad del sector en } \\
\text { el mercado mundial. Particularmente, los valores negativos mayores a dos destacan una } \\
\text { significativa dependencia por importaciones. }\end{array}$} \\
\hline
\end{tabular}

Fuente: United Nations Commodity Trade Statistics Database: http://comtrade.un.org/db/, consultado octubre 2, 2010.

\section{Conclusiones}

El déficit comercial, principal componente del saldo de la cuenta corriente estadounidense, se puede considerar, a través del efecto ingreso y tipo de cambio, el resultado de una política estratégica de Estados Unidos dirigida a financiar su crecimiento económico mediante ahorros foráneos. Hasta el 
primer trimestre del año 2007, el ahorro mundial, que resultó de los países en desarrollo de la región asiática, encontró un domicilio rentable en Estados Unidos por la condición del dólar como moneda líder de reserva internacional, el ciclo expansivo de crecimiento asociado al desarrollo del sector tecnológico e informático, y la fase de recuperación económica relacionada con la "bursatilización" del mercado inmobiliario. De forma tal que, mientras Japón y China adquieran significativas cantidades de valores financieros transados en el mercado de capital estadounidense, el financiamiento del DCEU es posible. Sin embargo, la dependencia crónica del capital foráneo parece encontrar un lado vulnerable en el largo plazo debido a las características de los instrumentos negociados en la actual fase de liberalización financiera.

Por otra parte, el estudio de los índices de ventaja comparativa revelada en el Este de Asia comprueba que la reestructuración industrial del Este de Asia, liderada por Japón, ha contribuido en ubicar a China como un líder exportador hacia el mercado de Estados Unidos. Existe, de hecho, una total relación entre la hegemonía china en el mercado estadounidense de eléctricos y electrónicos y las características de su plataforma exportadora de carácter regional, aún caracterizada por altos coeficientes de importación de partes y componentes, particularmente, en la fabricación de manufacturas de alto contenido tecnológico. En general, las causas de la participación de China en el DCEU están vinculadas con la operación de su plataforma exportadora, en la cual el capital estadounidense conserva una participación más que considerable.

Un conjunto de elementos asociados con los modelos de desarrollo económico vigentes en China y Estados Unidos se requiere analizar para alcanzar una visión integral de la participación de China en el DCEU. Por lo pronto, el desafío para Estados Unidos, entre otros elementos, parece ubicarse en alcanzar un conjunto de políticas de innovación tecnológicas que logren ubicar al mercado interno como fuerza primordial de un nuevo ciclo de crecimiento económico nacional.

\section{Bibliografía}

Arrighi, Giovanni (2010), The Long Twentieth Century. Money and Origins of our Times, Londres, Verso. pp. 336-370.

Athukorala, Prema-Chandra (2010), "China and Southeast Asia in the New Regional Division of Labour", en Yueh, Linda (ed.), The Future of Asian 
Trade and Growth. Economic Development with the Emergence of China, Londres, Routledge, pp. 406-432.

Blecker, Robert, A. (1996), U.S. Trade Policy and Global Growth, Washington, DC, Economic Policy Institute.

Bradsher, Keith (2008), "Investors Seek Asian Options to Costly China", The New York Times, Negocios Mundiales, 18 de junio, p. A1.

Elwell, Craig K. (2004), "Deindustrialization of the U.S. Economy: The Roles of Trade, Productivity, and Recession", CRS Report for Congress, RL32350. Griswold, Daniel T. (2001), “America's Record Trade Deficit. A Symbol of Economic Strength", Trade Policy Analysis, 12, pp. 1-19.

International Monetary Fund (2006), "Global Financial System Resilience in the face of Cyclical Challenges", Global Financial Stability Report, 1, p. 35. Krugman, Paul (2009), The Return of Depression Economics and the Crises of 2008, New York, W.W. Norton \& Company, pp. 56-76, 139-152.

L. Mann, Catherine (1999), Is the U.S. Trade Deficit Sustainable?, Washington, DC, Peterson Institute for International Economics.

National Center for Science and Engineering Statistics, NCSES (2012), "Industry, Technology, and the Global Marketplace", Science and Engineering Indicators 2012, Arlington, VA, National Science Board, pp. 1-74.

National Center for Science and Engineering Statistics (2010), "Industry, Technology, and the Global Marketplace", Science and Engineering Indicators 2010, Arlington, VA, National Science Board, pp. 2- 60.

Pakko, Michael P. (1999), "The U.S. Trade Deficit and the "New Economy", Review, septiembre - octubre, pp. 11-20.

Pérez, Carlota (2002), Revoluciones tecnológicas y capital financiero, México, Siglo XXI editores.

Ping, Xin-Qjao, "Vertical Specialization and Intra-Industry Trade in China (1992-2003)", en Linda Yueh (ed.), The Future of Asian Trade and Growth. Economic Development with the Emergence of China, Londres, Routledge, pp. 75-90.

Thomas L. Vollraht (1991), "A Theoretical Evaluation of Alternative Trade Intensity Measures of Revealed Comparative Advantage", Review of World Economics, 2(127), pp. 265-279.

United States Census Bureau (2009), "The 2009 Statistical Abstract: PDF Version", Washington, DC, U.S. Department of Commerce, pp. 787, 796. United States Government (2008), Economic Report of the President, Washington, DC, United States Government Printing Office, p. 353. 


\section{Fuentes electrónicas}

Bernanke, Ben S. (2005), "The Global Saving Glut and the U.S. Current Account Deficit", Sandridge Lecture, March 10. Disponible en: http://www. federalreserve.gov/boarddocs/speeches/2005/200503102/. Consultado: noviembre 18, 2008.

Chen, Xi Kong; Leonard K. Cheng; K. C. Fung y Lawrence J. Lau (2007), The Estimation of Domestic Value-Added and Employment Induced by Exports: An Application to Chinese Exports to the United States. Disponible en: http:// www.bm.ust.hk/ ced/Bookchapter(Final).pdf. Consultado: diciembre 18, 2007.

CNNEXPANSIÓN.com (2008), Hanesbrands cerrará planta en México. Disponible en http://www.cnnexpansion.com/negocios/2008/09/25/hanesbrandscerrara-planta-en-mexico. Consultado: abril 20, 2010.

\section{Banco de datos}

BEA, National Economic Accounts, www.bea.gov

BEA, International Economic Accounts, www.bea.gov/international/dilusdbal.htm

Cepal, Magic Plus, www.eclac.org/magic/demo.aspx?id=0

Foreign Trade Department of the Ministry of Commerce (MOFCOM), FDI Statistics, www.fdi.gov.cn/pub/FDI_EN/Statistics/AnnualStatisticsData/default.jsp FMI, World Economic Outlook Database, www.imf.org/external/pubs/ft/weo/2010/01/weodata/index.aspx U.S. Department of the Treasury, www.treasury,gov/resource-center/data-chart-center/tic/Pages/ticsec.aspx United Nations Commodity Trade Statistics Database, http,//comtrade. un.org/db/

World Bank Atlas, http,//data.worldbank.org/indicator/NE.EXP.GNFS.ZS 


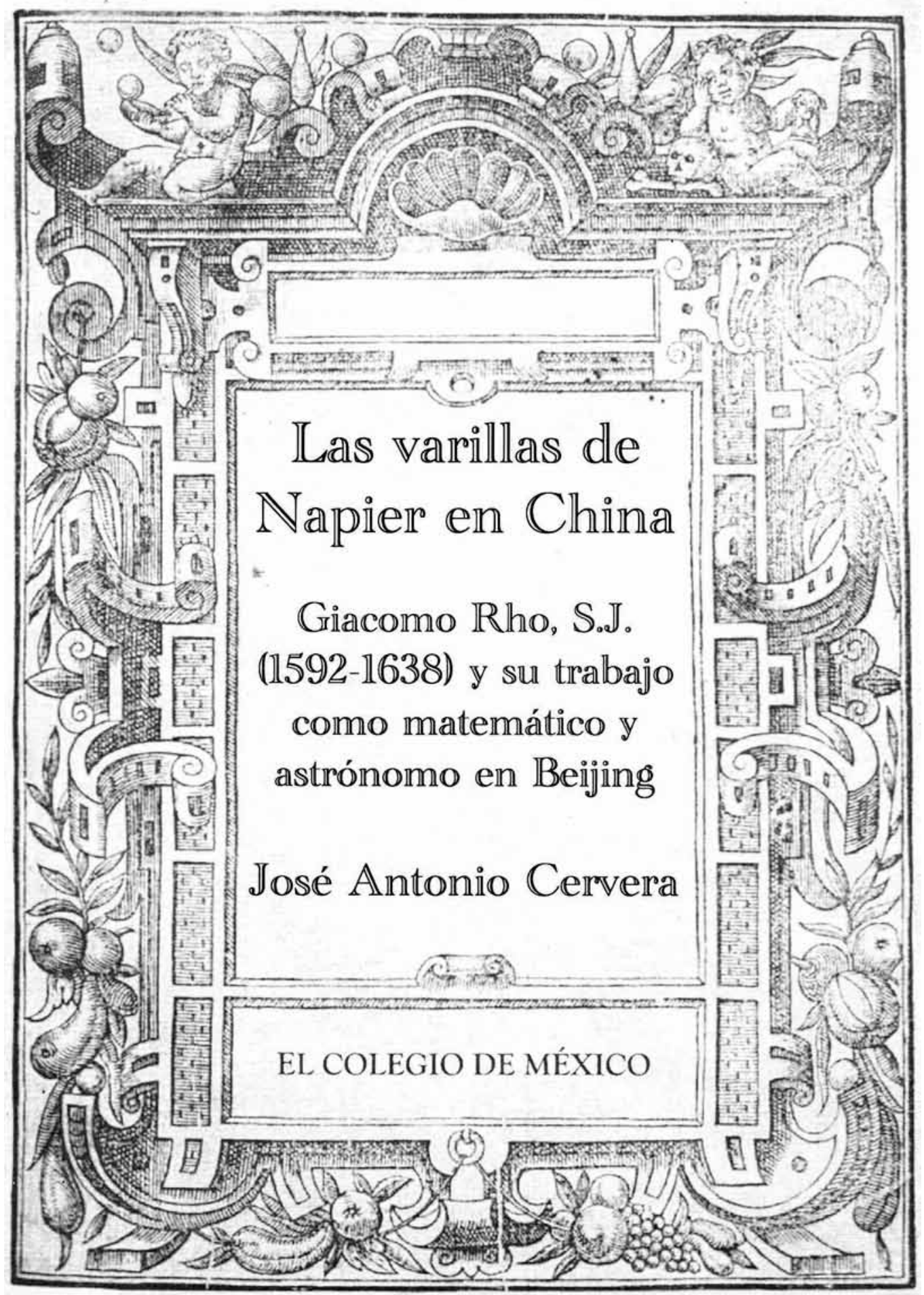

